\title{
Deutsche Europapolitik nach dem Regierungswechsel 2005
}

\author{
Hartmut Marhold*
}

\section{Europa - kein Wahlkampfthema}

Im Bundestagswahlkampf hat die Europapolitik eine untergeordnete Rolle gespielt, Anzeichen für einen tiefgreifenden Politikwechsel hat es nicht gegeben. Das liegt zum einen sicher an einem deutschen Spezifikum: Europa ist kein Streitfall zwischen den Parteien, der Konsens darüber, dass die europäische Integration zur deutschen Staatsräson gehört, reicht weit und lässt Divergenzen allenfalls in konkreten Einzelfällen oder Stilfragen zu. ${ }^{1}$ Zum anderen war die Abwesenheit von Europa-Themen im Wahlkampf auf die Lage der Europäischen Union selbst zurückzuführen, auf die ,Reflexionsphase', die die Staats- und Regierungschefs nach den gescheiterten Verfassungsreferenden in Frankreich und den Niederlanden bei ihrem Juni-Gipfel verordnet hatten. Daneben war im Juni auch die Einigung über die mittelfristige Finanzplanung der Union für die Jahre 2007 bis 2013 gescheitert, ein neuer Kompromiss nicht in Sicht. Einige Monate zuvor schien es gelegentlich, als solle das Verhältnis zwischen der Europäischen Union und der Türkei ein Wahlkampfthema werden, aber die Entscheidung, am 3. Oktober Verhandlungen aufzunehmen, war im Frühjahr längst gefallen (auch wenn die Zypern-Frage noch zu einem Stein des Anstoßes hätte werden können). Schließlich ist die französische Politik schon jetzt durch die Infragestellung der Autorität Jacques Chiracs als Staatspräsident und die Aussichten auf die Wahlen 2007 blockiert, in Großbritannien reicht die Zukunft Tony Blairs nicht mehr sehr weit, in Polen standen ebenfalls für den Herbst 2005 Neuwahlen an - so dass insgesamt die europäische Politik ein unübersichtliches Terrain war und ist, auf dem Blockade, Irritation und Orientierungssuche keine idealen Voraussetzungen für wahlkampfwirksame Positionierungen abgaben.

Bei einer tiefergehenden Analyse stellt sich heraus, dass die Konzentration auf innenpolitische Themen, auf die Reform des Sozialstaates, auf die Wiederbelebung von Wirtschaftswachstum nur scheinbar von Europa absieht: Gerade diese Aufgaben haben, jedenfalls in einem so großen Teilbereich des Europäischen Binnenmarktes wie der deutschen Volkswirtschaft, selbst erhebliche Bedeutung für Europa, für die gemeinsame Währung und für die Finanzierung europäischer Politik. Insofern war und ist die Fokussierung auf Wirtschaftswachstum und Sozialstaatsreformen implizit auch eine europäische Aufgabe für deutsche Politik - viele andere Mitgliedstaaten warten sehnsüchtig auf eine Wiederbelebung der deutschen Wirtschaft, im eigenen und im europäischen Interesse. Im deutschen Wahlkampf den Blick auf die innerstaatlichen Wachstums- und Sozialprobleme zu richten, entspricht durchaus der geltenden Arbeitsteilung zwischen Europäischer Union und Mitgliedstaaten, der zufolge die Union für die Organisation des Binnenmarktes, die Mitgliedstaaten dagegen für Sozialpolitik und für einen Großteil der Wachstumsbedingungen zuständig sind. In dieser Hinsicht ist Europa im deutschen Wahlkampf implizit öfter als auf den ersten Blick erkennbar, und zwar als Rahmenbedingung, unter der die deutschen Regeln für Arbeitsmarkt, Sozialversicherungen, Steuern et cetera neu zu gestalten wären. Andererseits freilich kann

* Dr. Hartmut Marhold, Generaldirektor, Centre International de Formation Européenne, Nizza.

1 Vgl. allgemein zur unmittelbaren Vorgeschichte der hier geschilderten Entwicklungen Josef Janning: Bundesrepublik Deutschland, in: Werner Weidenfeld/Wolfgang Wessels (Hrsg.): Jahrbuch der Europäischen Integration 2005, Baden-Baden 2005, S. 309-316. 
man bedauern, dass ein so wichtiges Instrument der Politikgestaltungsfähigkeit wie Europa nicht in die Debatte um die Zukunftsfähigkeit Deutschlands einbezogen worden ist. Viele, auch sozialstaatliche und wachstumsrelevante Faktoren entgehen mehr und mehr nationaler ,governance capacity', die Gestaltungsfähigkeit auf diesen Gebieten kann allenfalls auf europäischer Ebene wiedererlangt werden, angesichts europaweiter gemeinsamer Herausforderungen wie der demografischen, globaler Herausforderungen wie der wirtschaftlichen Integration der Weltmärkte oder der weltweiten Sicherheitsprobleme. In dieser Hinsicht wurde im Wahlkampf von allen Seiten die Illusion genährt, man könne die in Deutschland auftretenden Probleme mit Mitteln der deutschen Bundespolitik lösen. Ob unter diesen veränderten Umständen die oben angedeutete Arbeitsteilung innerhalb des europäischen Mehrebenensystems noch tragfähig ist, wird intensiv diskutiert, etwa am Beispiel des ,Europäischen Gesellschaftsmodells', wie das informelle Treffen des Europäischen Rats in Hampton Court im Oktober 2005 zeigte.

Auf der anderen Seite waren durchaus unterschiedliche Positionen zwischen den späteren Koalitionspartnern erkennbar gewesen, auch wenn diese zum Teil nur auf die Rollenverteilung zwischen Regierung und Opposition zurückzuführen waren, wie etwa im Falle der Neuverhandlung der Dienstleistungs-Richtlinie. Noch bei ihrer Rede zur Abstimmung des Bundestages über den Europäischen Verfassungs-Vertrag hatte Angela Merkel - als Oppositionsführerin - diese Richtlinie als ,,von Grund auf richtig“ bezeichnet und kritisiert, dass man ,plötzlich in Situationen, in denen es zu bestimmten Entscheidungen kommt, etwas nuanciert und anders" darstelle, ,,als man das vorher getan hat". 2

Wie bereits angedeutet, waren auch die Auffassungen über das anzustrebende Verhältnis zwischen Europäischer Union und der Türkei durchaus umstritten. Während die SchröderRegierung, im Schulterschluss mit Jacques Chirac, eine Vollmitgliedschaft entschieden befürwortete und aktiv für die Aufnahme von Beitrittsverhandlungen mit diesem Ziel eintrat, hatten sich CDU und CSU für eine Alternative zur Mitgliedschaft, für eine ,Privilegierte Partnerschaft ${ }^{\star}$, stark gemacht. Auch im Verhältnis zu anderen europäischen Partnern, vor allem zu den ,kleineren' EU-Mitgliedstaaten, hatten CDU und CSU eine andere Politik gewünscht, Kritik an der ,Mittelmacht'- oder ,Zentralmacht'-Vorstellung ${ }^{3}$ geübt, an Vorstellungen von einem ,deutschen Weg“, ${ }^{4}$ an der „Achsenbildung“5 zwischen Paris, Berlin und Moskau, die von anderen EU-Mitgliedern als Affront und Missachtung empfunden wurde. Dieser Aspekt divergierender Auffassungen leitet über zu Stilfragen im Umgang mit den

2 Angela Merkel: Ja zum Vertrag über die Verfassung in Europa. Rede zur EU-Verfassung im Deutschen Bundestag, 12. Mai 2005, vgl. http://www.cducsu.de/section_1/subsection_5/id_1530/Meldungen.aspx (letzter Zugriff: 15.11.05). Vgl. auch die ausführliche Hintergrundanalyse von Isabelle Bourgeois: Directive „Services": les enjeux du débat allemand, in: Regards sur l'économie allemande Nr. 71/2005, S. 5-14.

3 Vgl. zu diesen und weiteren Begriffen von deutscher ,-macht“ u.a. Wolfgang Wessels: Zentralmacht, Zivilmacht oder Ohnmacht? Zur deutschen Außen- und Europapolitik nach 1989, in: Peter R. Weilemann, Hans Jürgen Küsters, Günter Buchstab (Hrsg.): Macht und Zeitkritik. Festschrift für Hans-Peter Schwarz zum 65. Geburtstag. Paderborn, München, Wien, Zürich 1999, S. 299-406; in neuerer Zeit die Kontroverse zwischen Hans-Peter Schwarz und Hanns W. Maull, vgl. Hans-Peter Schwarz: Republik ohne Kompass. Anmerkungen zur deutschen Außenpolitik. Berlin 2005, S. 17 bzw. S. 314.

4 Vgl. zum Begriff „Deutscher Weg“ Klaus Hartung: „Der neue deutsche Weg“, in: Die Zeit, Nr. 42/2003 (09.10.2003). Während Hartung den Begriff innenpolitisch wendet (als spezifisch deutsche Variante von Sozialstaatsreform), analysiert Christian Hacke die außenpolitischen Dimension: Die Außenpolitik der Regierung Schröder/Fischer: Zwischenbilanz und Perspektiven, in: APuZ 48/2002, S. 7-15. Hackes Analyse wird fortgeführt von Gunther Hellmann: Von Gipfelstürmern und Gratwanderern: „Deutsche Wege“ in der Außenpolitik, in: APuZ 11/2004, S. 32-39.

5 Vgl. „Bricht die Achse Paris-Berlin-Moskau?“, in: Die Welt, 20.07.2005. Die ,Achsen“-Debatte in dieser Konstellation war besonders virulent in den Jahren 2001 bis 2003, zumal angesichts der Gegnerschaft der drei Staaten gegen den Irak-Krieg. 
EU-Partnern, in denen Regierung und Opposition immer wieder unterschiedliche Herangehensweisen erkennen ließen. Schließlich waren deutliche Unterschiede auch im Hinblick auf das Verhältnis zu den Vereinigten Staaten erkennbar, auch wenn die Einstellungen nicht so konträr geblieben waren wie zur Zeit des Irak-Krieges, als Gerhard Schröder aus eigenem Entschluss den USA die Gefolgschaft aufkündigte, während Angela Merkel Bündnispflichten auch in diesem kritischen Fall konsequent wahrzunehmen bereit gewesen wäre. Inzwischen hatte sowohl die Regierung Schröder wieder Schritte zur Annäherung an die USA unternommen (und auf der Arbeitsebene war ohnehin die Kooperation intensiv), wie auch CDU/CSU von ihrer Bereitschaft, den USA selbst in den Irak zu folgen, längst abgerückt waren.

Alles in allem ließ sich auf Grund des Bundestagswahlkampfes ein hohes Maß an Kontinuität in der deutschen Europapolitik erwarten, wenn man die Abwesenheit von europäischen Themen, den Grundkonsens zwischen den Parteien über Europa, die europapolitische Lage selbst und die wenigen erkennbaren Divergenzen zum Maßstab nimmt.

\section{Auf dem Weg zur Großen Koalition - Kontinuitätserwartungen bestätigen sich}

\section{Von Schröder zu Merkel}

Der Wahlausgang und der Weg zur Großen Koalition konnten diese Erwartungen nur bestärken. Zwar löste eine CDU-geführte eine SPD-dominierte Regierung ab, eine Kanzlerin Merkel einen Kanzler Schröder - und beider politisches und persönliches Profil ist zweifellos ganz unterschiedlich, mit entsprechenden Auswirkungen auch auf die Europapolitik. Von der neuen Kanzlerin aus dem Nordosten der früheren DDR, mit einem Erfahrungshorizont, der sich vor 1989 nach Osten, nach Polen, in die Tschechoslowakei (wo sie studierte) und - obligatorisch - in die Sowjetunion öffnete, wird man trotz aller seitdem hinzugewonnenen politischen Expertise ein besonderes Verständnis für die neuen EU-Mitgliedstaaten, für Transformationsprozesse und für kleinere EU-Partner erwarten. Dass Angela Merkel schon vor den Bundestagswahlen auf europäischer Bühne ungewöhnlich aktiv und präsent war, dämpfte erheblich die Befürchtung, es werde erst wieder eine längere Lernphase auf der europäischen Szene geben. Die beiden in der Öffentlichkeit wohl am deutlichsten wahrgenommenen Themen, bei denen die CDU-Vorsitzende europäisches Profil gewann, waren einerseits die Türkei-Frage, in der sie die europäischen Christdemokraten in der Europäischen Volkspartei (EVP) stark beeinflusste, und andererseits die Auswahl des amtierenden Kommissionspräsidenten: Mit keinem anderen als jemandem aus dem eigenen Lager wollte sich Angela Merkel nach dem Wahlsieg der EVP bei den letzten Europawahlen zufrieden geben, Barroso war vor allem ihre Wahl. Durch europaskeptische Positionen war sie dagegen nicht aufgefallen, im Gegensatz zu ihrem Vorgänger, der vor seinem Amtsantritt noch als Zweifler am Nutzen der Währungsunion galt, sich dann aber über mehrere Zwischenschritte und Umwege zum engagierten ,Europäer' wandelte - der Weg von seinen zuletzt vertretenen Ansichten zu Angela Merkels ersten europapolitischen Positionsbestimmungen ist dann nicht mehr weit gewesen, ${ }^{6}$ so dass es wenig überraschen konnte, den Noch-Bundes-

6 Übereinstimmungen sind etwa mit Ausgleich, Wettbewerb - Soziale Marktwirtschaft, Angewiesenheit Deutschlands auf Europas Gestaltungsfähigkeit in der globalisierten Welt, Begrenzung der EU-Gesetzgebung auf Kernkompetenzen, Wertorientierung der europäischen Integration vorhanden, wie ein Vergleich zwischen Schröders Beitrag unter dem etwas überraschenden Titel: „Auf die Kleinen ist Verlass“, in: Die Zeit, Nr. 43/ 2005, 20.10.2005 und dem Interview mit Angela Merkel eine Woche später zeigt: „Merkel drängt in der EUFinanzpolitik auf Sparsamkeit“, in: Handelsblatt, 27.10.2005. Vgl. dagegen Hackes Rede von „Fischers postnationaler Einstellung zur Außenpolitik“, Hacke: Die Außenpolitik der Regierung Schröder/Fischer. 
kanzler zum Sozialgipfel nach Hampton Court zu schicken, um ihn dort gegenüber den britischen Vorschlägen Auffassungen vertreten zu lassen, mit denen Angela Merkel übereinstimmen konnte.

\section{Von Fischer zu Steinmeier}

Kontinuität verheißt auch der Verbleib der SPD in der Regierung, zumal sie nicht nur durch die Partei, sondern auch durch Ämter und Personen verbürgt wird. Das gilt insbesondere für den neuen Außenminister Frank-Walter Steinmeier, der schon in Niedersachsen zu Gerhard Schröders Team gehörte, dann in seiner Regierung als Kanzleramtsminister fungierte und in dieser Eigenschaft für die Kohärenz der gesamten Politik der Bundesregierung zuständig war - eine Aufgabe, die ihn zweifellos auch als Außenminister prägt. Der Unterschied zu seinem Vorgänger Joschka Fischer ist weniger in anderen außenpolitischen Optionen, als vielmehr in einem Paradigmenwechsel von Außenpolitik überhaupt gesehen worden. Während Fischer als einer der letzten Vertreter, klassischer' Außenpolitik gedeutet wurde, glauben manche Kommentatoren in Steinmeier den ersten Anwalt „postklassischer“, das heißt mit anderen Politiken integrierter, vernetzter Außenpolitik sehen zu können. ${ }^{7}$ Das ist etwas anderes und weit mehr als nur ein administratives, bürokratisches Verständnis von Außenpolitik und lässt sich auch nicht auf reinen Pragmatismus reduzieren. Vielmehr prädisponiert eine solche (Außen-)Politikdefinition gerade zum Verständnis des komplexen europäischen Mehrebenen-Systems, eines föderalen Integrations- und Kooperationsverbundes, in dem die Unterscheidung zwischen Innen- und Außenpolitik keine große Relevanz mehr hat, jedenfalls nicht unter den Mitgliedstaaten. ${ }^{8}$ Wenn es dieses Profil ist, das der neue AuBenminister pflegt und entwickelt, dann wird keine Fremdheit in der Europapolitik auftreten, und selbst die Rolle des Auswärtigen Amtes als Haupt-Koordinator der deutschen Europapolitik kann davon profitieren. Insoweit dagegen die Schrödersche Außen- und Europapolitik vom besonderen persönlichen Stil des früheren Bundeskanzlers geprägt war - und mutatis mutandis gilt das auch vom früheren Außenminister Fischer -, lässt sie sich ohnehin kaum von einem anderen fortsetzen.

\section{Pacta sunt servanda - Kontinuität, auch im Falle der Türkei-Frage}

Kontinuität ist auch das entscheidende Merkmal der Koalitionsverhandlungen gewesen, soweit sie die Europapolitik betreffen und sich im Koalitionsvertrag niederschlagen. ,Pacta sunt servanda" ist die Regel, die von Angela Merkel selbst auf den Gebieten akzeptiert worden ist, auf denen die früheren Positionen voneinander abweichen. Auf die Frage eines Journalisten, ob ,das Thema der türkischen EU-Beitrittsverhandlungen zur Sollbruchstelle der großen Koalition“ werde, antwortete sie: „Nein. Die Entscheidung ist gefallen, dass die EU mit der Türkei verhandelt. Wir werden uns jetzt nach dem Prinzip ,Pacta sunt servanda’ auf die Verhandlungen einrichten [...] Unabhängig davon halten wir als Union an dem Ziel der privilegierten Partnerschaft der EU mit der Türkei fest“. .9 Ob die Formel, die der Koalitions-

7 Vgl. seine Rede zur Verabschiedung von Christoph Bertram bei der SWP am 21.9. 2005, http://www.swp-ber lin.org/common/get_document.php?id=1428 (letzter Zugriff: 06.01.2005). Vgl. zu Steinmeier auch: „An der Seite der Kanzler“, in: Der Tagesspiegel, 14.10.2005, S. 3. Vgl. auch „Das Vorstellungsgespräch“, in: Die Zeit, 49/2005, 1. Dezember 2005, S. 4. Dort heißt es u.a.: „Fischer wollte klassische Machtpolitik betreiben. Steinmeier ist postklassisch“, was heißen soll, dass er „,ie Außenpolitik mit offenen Rändern zur Sicherheits- und Entwicklungspolitik“ sieht. Als Bildunterschrift findet sich hier die Formel „Arbeitsminister des Äußeren“.

8 Vgl. „Das Vorstellungsgespräch“, in: Die Zeit, Nr. 49/2005, S. 3: „Das Postklassische muss Steinmeier auch zum Europa-Geschäft ziehen, wo die Grenzen von Außen- und Innenpolitik verschwimmen und die Wirkungen von Kommissionsrichtlinien auf die heimische Chemieindustrie zu prüfen sind“". Angela Merkel, Interview im Handelsblatt, 27.10.2005. 
vertrag enthält - dort ist nicht von ,privilegierter Partnerschaft', sondern (,,sollte die EU nicht aufnahmefähig oder die Türkei nicht in der Lage sein, alle mit einer Mitgliedschaft verbundenen Verpflichtungen voll und ganz einzuhalten“) von einem ,,privilegiertem Verhältnis" "10 die Rede - genau das gleiche meint, oder ob zwischen ,Verhältnis “ und ,Partnerschaft' Nuancen bestehen, lässt sich kaum erkennen. Bei einer Analyse mit diesem Detailgrad ist denn auch daran zu erinnern, dass der neue Kanzleramtsminister de Maizière generell zum Koalitionsvertrag anzumerken hatte, er sei „,keine Bibel“.11

\section{Europäischer Verfassungsvertrag}

Bei den meisten anderen Themen ist die zu vermutende Kontinuität noch ausgeprägter. Das gilt für die Zukunft des Europäischen Verfassungsvertrages, der ohnehin nicht das Werk einer Partei oder Regierung, sondern ein Konsensunternehmen europäischer Dimension par excellence war. Dementsprechend hatte Angela Merkel auch bereits in ihrer Rede vom 12. Mai, anlässlich der Bundestagsabstimmung über die europäische Verfassung, weit gehende Übereinstimmung zum Ausdruck gebracht - und bei dieser Gelegenheit ihre europapolitische Marschrichtung verdeutlicht -, bestätigt durch eine parteiübergreifende Mehrheit bei der dann folgenden Abstimmung. Aber auch in der Reaktion auf die Ablehnung des Verfassungsvertrages bei den Volksabstimmungen in Frankreich und den Niederlanden am 29. Mai beziehungsweise am 2. Juni waren sich Regierung und Opposition einig und konnten relativ mühelos zur gemeinsamen Festlegung im Koalitionsvertrag gelangen: „Wir stehen zum Europäischen Verfassungsvertrag. [...] Deshalb treten wir dafür ein, die Ratifizierung [...] fortzuführen und unter deutscher Präsidentschaft im ersten Halbjahr 2007 neue Anstöße zu geben. "12 Darin kommt neben dem Kontinuitätsbekenntnis auch die Einsicht zum Zuge, dass die Bestimmung des Verfassungsvertrages selbst, der zufolge der Europäische Rat mit dem Problem der Ratifizierung ,befasst wird, wenn nach Ablauf von zwei Jahren nach der Unterzeichnung des Vertrages [...] Schwierigkeiten [...] aufgetreten sind“, 13 nicht mehr der aktuellen Lage gerecht wird. Zudem wird an dieser Stelle wie an vielen anderen im europapolitischen Teil des Koalitionsvertrages deutlich, dass sich die Erwartung einer neuen europapolitischen Dynamik an die deutsche Ratspräsidentschaft im ersten Halbjahr 2007 knüpfen, und dies nicht nur aus Gründen, die in Deutschland liegen, wie bereits angemerkt wurde. Das Festhalten am Ratifizierungsziel ${ }^{14}$ hindert die Große Koalition nicht, wie sich noch auf einzelnen Politikfeldern zeigen wird, für die Anwendung bestimmter Be-

10 Koalitionsvertrag zwischen CDU, CSU und SPD, Gemeinsam für Deutschland - mit Mut und Menschlichkeit, 11.11.2005 (zu Europa und Außenpolitik S. 126-140), S. 130; siehe auch http://www.zeit.de/online/2005/46/ eckpunkte und http://www.cducsu.de/upload/2C2581D5821FD61A7A4DEA71E3C644CA11376-by1b0oli.pdf (letzter Zugriff: 14.11.2005).

11 Volkery/Weiland zitieren Äußerungen des Kanzleramtsministers aus einer Talkshow: „Man solle den Text nicht überbewerten, wenn er Ende der Woche stehe, so der Vertraute der künftigen Kanzlerin Angela Merkel. ,Der Koalitionsvertrag ist keine Bibel', betonte de Maizière. Da entstehe ,eine Scheinplanung über vier Jahre', die später gar nicht so eintreten werde. Die Wirklichkeit sei ,viel intelligenter', als das, was aufgeschrieben werde.“ Carsten Volkery/Severin Weiland: „Koalitionsvertrag ist keine Bibel“, abrufbar unter http:// www.spiegel.de/politik/deutschland/0,1518,384233,00.html (letzter Zugriff: 21.12.2005). Nebenbei sei angemerkt, worauf Helmut Kohl in seinem gerade erschienenen zweiten Memoirenband hinweist: Im Unterschied zu den 191 Seiten des gegenwärtigen hatte der Koalitionsvertrag von 1983 nur sechs Seiten, Helmut Kohl: Erinnerungen 1982-1990, München 2005, S. 116.

12 Koalitionsvertrag, S. 127.

13 Vertrag über eine Verfassung für Europa (VVE), Europäische Gemeinschaften 2005, Erklärung Nr. 30 der Schlussakte, S. 472.

14 Zumindest der Hinweis ist hier vonnöten, dass auch in Deutschland der Verfassungsvertrag noch nicht ratifiziert ist - erst die Unterschrift des Bundespräsidenten beendet das Verfahren, und der wartet bekanntlich auf ein Urteil des Bundesverfassungsgerichtshofes, nach einer Klage gegen die Rechtmäßigkeit des VVE. 
stimmungen „schon vor Inkrafttreten des Verfassungsvertrages“ einzutreten, so etwa für „,die Anwendung des Subsidiaritäts-Frühwarnsystems [...], wozu es keiner Vertragsänderung bedarf" und das die frühere Einbindung der nationalen Parlamente in den europäischen Gesetzgebungsprozess sichern soll. ${ }^{15}$

Trotz dieser integrationsprogressiven Festlegung auf das Verfassungsziel ähneln sich die Aussagen des ehemaligen Bundeskanzlers und der Bundeskanzlerin auch zur Integrationsbegrenzung. Hatte Schröder noch zuletzt von der ,Überdehnung der europäischen Kompetenzen“, von der Gefahr eines ,schleichenden Souveränitätsverlusts“ gesprochen, von ,europäischen Regelungen [...], die gar nicht erforderlich sind“" (und frühere Beispiele für seine entschiedene Opposition zur Europäischen Kommission lassen sich leicht erinnern), so verlangte Angela Merkel schon in ihrer Bundestagsrede vom 16. Juni eine Antwort auf die Frage, ,welche politische Selbstbeschränkung sich Europa auferlegt“, eine „Notwendigkeitskontrolle“, bekundete Verständnis für die „Sorgen“ der Bürger, ,wenn sie hören, dass es 400, 500 oder 600 neue Richtlinien geben könnte“, und forderte „eine politische Kontrolle dessen [...], was in der Kommission an Richtlinien verabschiedet wird."16 Angesichts dieses Gleichklangs wird es nicht schwierig gewesen sein, in den Koalitionsvertrag die Forderungen einzubauen, ,unnötige Bürokratie abzubauen und die europäische Gesetzgebung auf das tatsächlich Notwendige zu beschränken [...], um einer Aushöhlung der Zuständigkeiten der Mitgliedstaaten vorzubeugen“. Die Skepsis gegenüber der Kommission reicht hier bis zu der Auffassung, der Europäische Rat müsse ,im Einzelfall von seinem Recht Gebrauch machen, die Kommission aufzufordern, einen Gesetzgebungsvorschlag zurückzuziehen". 17

\section{Haushalt, Finanzen, Stabilitätspakt}

Zwar hatte die Opposition die Bemühungen der Schröder-Regierung um eine Neufassung des Stabilitäts- und Wachstumspakts heftig kritisiert, aber die große Koalition hat sich schnell darauf geeinigt, an der im März unter luxemburgischer Ratspräsidentschaft reformierten Version festzuhalten und das Neuverschuldungs-Ziel nach dann vier Jahren Verfehlung 2007 wieder zu erreichen - also nicht bereits 2006, was angesichts der kurzen Zeit, die zur Sanierung der deutschen öffentlichen Haushalte dann nur zur Verfügung stünde, ein illusionäres Ziel gewesen wäre. Allerdings muss die Einhaltung des Stabilitätspaktes im Jahr 2007 durch Maßnahmen (Gesetzgebung wie Haushaltsvorbereitungen) in den ersten Monaten des Jahres 2006 vorbereitet werden, und in dieser Phase könnte es durchaus zu Konflikten zwischen den Koalitionspartnern sowie zwischen der Bundesregierung und der Europäischen Kommission kommen. So wie auch hier Kontinuität - jedenfalls generell hinsichtlich des Stabilitätspaktes ${ }^{18}$ - gilt, so in nicht weniger hohem Maß im Hinblick auf die mittelfris-

15 Vgl. zur möglichen Umsetzung außen-, sicherheits- und verteidigungspolitischer Elemente des VVE vor dessen Ratifizierung Christoph Heusgen: Nach den gescheiterten Referenden: Was wird aus dem Außenminister der Union und dem Europäischen Auswärtigen Dienst?, in: integration 4/05, S. 336-340.

16 Angela Merkel: Ein einfaches ,Weiter so' wird Europa zerstören. Rede zur Regierungserklärung des Bundeskanzlers, 16. Juni 2005; vgl. http://www.cducsu.de/section_1/subsection_6/id_3601/Meldungen.aspx (letzter Zugriff: 15.11.2005).

17 Koalitionsvertrag, S. 127. In ihrer Rede vom 12. Mai zum Verfassungsvertrag hatte Angela Merkel auch ein Thema aus dem letzten Wahlkampf wieder aufgenommen - damals von Edmund Stoiber und Wolfgang Clement in parteiübergreifender Einigkeit vorgetragen -, indem sie „Diskussionen“ darüber anmahnte, ,ob nicht Kompetenzen, die Europa schon einmal hatte und die in dem viel gelobten Acquis communataire verankert sind, wieder an die Nationalstaaten zurückgegeben werden." Angela Merkel: Ja zum Vertrag über die Verfassung in Europa, Rede zur EU-Verfassung, 12.05.2005, vgl. http://www.cducsu.de/section_1/subsection_6/ id_3467/Meldungen.aspx (letzter Zugriff: 15.11.2005).

18 Vgl. „Berlin wehrt sich gegen schärferes Defizitverfahren“, in: FAZ 05.12.2005, S. 1. 
tige Finanzplanung der Union für die Jahre 2007 bis 2013. Die deutsche Position war von der Regierung Schröder bereits im Jahr 2003 festgelegt worden, als die sechs ,Nettozahler * in einem gemeinsamen Schreiben die Begrenzung des EU-Haushalts auf 1 Prozent des BIP der Mitgliedstaaten gefordert hatten. Diese Forderung hatte sich die Opposition schon vor den Wahlen zu eigen gemacht, im gemeinsamen Widerspruch gegen Europäische Kommission und Europäisches Parlament, die deutlich über diese Schwelle hinauszugehen wünschten. In ihrer Rede zur Regierungserklärung des Bundeskanzlers nach der Tagung des Europäischen Rates kritisierte Angela Merkel am 16. Juni die Vorschläge des Europäischen Parlaments (Böge-Bericht) ${ }^{19}$ und wies darauf hin, dass Regierung und Opposition - „ob Sozialdemokraten oder Christdemokraten“ - in der Frage der Haushaltsplanung für die Europäische Union „die gleiche Perspektive“ einnähmen und sich „hier nicht vorwerfen“ sollten, was die Europaabgeordneten aller Parteien für weiter gehende Pläne entwerfen. Zwei Tage zuvor hatte Peter Hintze, europapolitischer Sprecher der CDU/CSU-Fraktion, in einem Interview Gerhard Schröder beim bevorstehenden Gipfel die Unterstützung der Union für das Ziel zugesagt, ,eine für Deutschland belastungsneutrale Lösung in der Nähe von ein Prozent des BIP“ zu erreichen: „Zuviel darüber kann er nicht gehen.“20 Bekanntlich wurde beim Juni-Gipfel eine Einigung nicht erreicht, und die Frage, wie viel über einem Prozent eine für Deutschland akzeptable Lösung liegt damit unbeantwortet gelassen. Immerhin hatte Gerhard Schröder, indem er die strikte ,1-Prozent-Position“ der sechs Nettozahler aufgegeben und Jean-Claude Juncker für seinen bei 1,06 Prozent liegenden Kompromissvorschlag Zustimmung signalisiert hatte, eine Verhandlungsmarge und -dynamik zugelassen, die Angela Merkel dann nutzen konnte.

\section{Binnenmarkt, Dienstleistungsrichtlinie, Agrarpolitik}

Die Koalitionsverhandlungen haben auch frühere Divergenzen in Sachen Dienstleistungsrichtlinie und Agrarpolitik glätten können. Nun ist von der Notwendigkeit die Rede, die vorgesehene Liberalisierung des Dienstleistungssektors - ein entscheidender Baustein des Binnenmarktes - mit Rücksicht auf deutsche Branchen-Interessen neu zu gestalten, dabei sollen ,,nicht zu rechtfertigende Hürden für deutsche Dienstleister im europäischen Ausland“ sowie ,das Herkunftslandprinzip noch einmal auf den Prüfstand“, wie Angela Merkel es im Interview formulierte. ${ }^{21}$ Allerdings wartet die Europapolitik nicht immer auf Deutschland: Im November 2005 hat der zuständige Ausschuss des Europäischen Parlaments die fragliche Richtlinie in einer überarbeiteten Fassung angenommen, das Mitentscheidungsverfahren geht seinen Weg. In ähnlicher Weise schwenkt die neue Regierung in der Agrarpolitik auf Kontinuitätskurs ein. Auch hier ist der gleiche Mechanismus des Wandels von einem behutsam-kritischen Oppositionskurs zur Fortsetzung der Regierungsposition am Werk: Wandel ist nur von der Opposition zur Regierung erkennbar, in der Haltung der aufeinander-

19 Vgl. zum Böge-Bericht die ausführliche Synthese und Stellungnahme des EP-Präsidenten Josep Borrell: Josep BORRELL speaking about the financial perspective: „The Böge report aims to be both ambitious and realistic"; http://www.europarl.eu.int/omk/sipade3?SAME_LEVEL=1\&LEVEL=4\&NAV=X\&DETAIL=\&PUB $\mathrm{REF}=-/ / \mathrm{EP} / / \mathrm{TEXT}+\mathrm{PRESS}+\mathrm{NR}-20050511-1+0+\mathrm{DOC}+\mathrm{XML}+\mathrm{V} 0 / / \mathrm{EN}$ (letzter Zugriff: 05.01.2006); ein kurzes Resümee des Zahlenwerkes im Böge-Bericht unter http://www.robert-schuman.org/lettre/lettreal212. htm\#26 (05.01.2006). Der Bericht selbst (vom 11.05.2005) unter http://www.europarl.eu.int/omk/sipade3?PU$\mathrm{BREF}=-/ / \mathrm{EP} / / \mathrm{NONSGML+REPORT+A6-2005-0090+0+DOC+WORD+V0//EN \& L=EN \& LE-}$

$\mathrm{VEL}=1 \& N A V=S \& L S T D O C=Y$ (letzter Zugriff: 05.01.2006).

20 Peter Hintze: EU-Finanzen - Hintze fordert belastungsneutrale Lösung in der Nähe von ein Prozent des BIP. Interview mit DeutschlandRadio Kultur, vgl. http://www.cducsu.de/section_1/subsection_5/id_1494/Mel dungen.aspx (letzter Zugriff: 15.11.2005)

21 Angela Merkel, Interview im Handelsblatt, 27.10.2005. 
folgenden Regierungen selbst dagegen dominiert Kontinuität. Als es im Juni um die mittelfristige Finanzplanung der Europäischen Union ging, hatte Angela Merkel noch den Britenrabatt und die Festlegung in der Agrarpolitik gleichermaßen für überholungsbedürftig erklärt - und damit implizit von Frankreich verlangt, den entscheidenden Schritt zur nationalen Kofinanzierung der Agrarpolitik zu tun: „Es kann nicht sein - das ist der Punkt -, dass von der einen Seite Flexibilität erwartet wird und von der anderen Seite gesagt werden kann: Für uns sind diese Dinge sakrosankt."22 Vor ihrem Regierungsantritt hatte die designierte Kanzlerin sich zwar immer noch gewünscht, ,eine verpflichtende nationale Kofinanzierung der EU-Agrarbeihilfen“ einzuführen, zugleich aber versichert: „Wir halten an dem Agrarfinanzkompromiss fest, er wird nicht noch einmal aufgeschnürt“".23 Und diese letztere Auffassung findet sich dann ohne weitere hoffnungsvolle Zusätze im Koalitionsvertrag wieder: „Die Zusagen aus dem Agrarfinanzkompromiss vom 24./25. Oktober werden nicht in Frage gestellt. Die Reform der Agrarpolitik wird auf dem eingeschlagenen Weg weitergeführt.“24

\section{Sozialpolitik, Lissabon-Strategie}

Die neue Bundeskanzlerin hat in ihren Reden und Interviews immer wieder einen Schwerpunkt auf die strategische Bedeutung der (ebenfalls im ersten Halbjahr 2005 unter luxemburgischer Ratspräsidentschaft reformierten) Lissabon-Strategie gelegt: „Diesem Ziel [der Lissabon-Agenda, nämlich ,zum dynamischsten wissensbasierten Wirtschaftsraum der Welt zu werden“] müsste die gesamte Gesetzgebungstätigkeit der Europäischen Union untergeordnet werden. “25 Das ist ein Leitmotiv für Angela Merkel, sie hat keine Gelegenheit ausgelassen, ihm Priorität zuzumessen: „Lasst uns politisch entscheiden, was wir im Sinne des Lissabon-Prozesses, also des Wachstumsprozesses, brauchen und was nicht! Lasst uns Prioritäten setzen!“26, so hieß es am 12. Mai in der Debatte über den Verfassungsvertrag, und fünf Wochen später: „Deshalb unterstütze ich alles, was mit der Lissabon-Strategie zusammenhängt.“27 Dementsprechend erfährt das Thema auch im Koalitionsvertrag eine besonders ausführliche Behandlung, die in dem Satz gipfelt: „Die Lissabon-Strategie bietet den geeigneten Rahmen für die wirtschaftliche und soziale Erneuerung in Europa." ${ }^{28}$ Vielleicht hier am ehesten lässt sich eine strategische Perspektive für die Europapolitik der neuen Bundesregierung erkennen, ein „Leitbild“, zumal die Rede von der Lissabon-Strategie immer wieder auch mit der Durchsetzung und dem Erhalt der Sozialen Marktwirtschaft auf europäischer Ebene in Verbindung gebracht wird. In ihrer Rede vom 12. Mai begründete Angela Merkel den Vorrang für Lissabon mit dieser Leitbild-Orientierung: „Es ist [...] von größter Bedeutung, dass es uns gelingt, Europa als Wertegemeinschaft, aber auch als ein Modell für das, was wir soziale Marktwirtschaft nennen, nämlich als Sozialstaatsmodell durchzusetzen“29, und am 16. Juni lautete ihr Credo: ,Wir sind einem Ziel verpflichtet, nämlich soziale Marktwirtschaft in Europa in Zeiten der Globalisierung durchzusetzen. " ${ }^{30}$ Fast die gleichen Formulierungen haben dann den Weg in den Koalitionsvertrag gefunden: „Die Durchsetzung der Grundsätze der Sozialen Marktwirtschaft auf europäischer Ebene wird nur

22 Angela Merkel, Rede vom 16.06.2005.

23 Angela Merkel, Interview im Handelsblatt, 27.10.2005.

24 Koalitionsvertrag, S. 128.

25 Angela Merkel, Interview im Handelsblatt, 27.10.2005.

26 Angela Merkel, Rede vom 12.05.2005.

27 Angela Merkel, Rede vom 16.06.2005.

28 Koalitionsvertrag, S. 128.

29 Angela Merkel, Rede vom 12.05.2005.

30 Angela Merkel, Rede vom 16.06.2005. 
gelingen, wenn wir unsere Wettbewerbsfähigkeit nachhaltig verbessern und zugleich den berechtigten Schutzbedürfnissen der Menschen Rechnung tragen." 31 Ob und wie sich aus solchen Prioritäten und ihrer Verknüpfung mit Zielvorstellungen in der Tat ein kohärentes und umfassendes Leitbild für die europäische Integration - im Sinne etwa eines „Europäischen Gesellschaftsmodells“ - als Perspektive deutscher Europapolitik entwickeln lassen, bleibt eine später zu erörternde Frage.

\section{Außen-, Sicherheits- und Verteidigungspolitik: Eine ,Gemeinsame‘ EU-Politik oder deutsche Sonderwege?}

Diskontinuitätsvermutungen gab es im Vorfeld der Regierungsbildung insbesondere auf dem Feld der Außenpolitik. Von einer CDU-geführten Bundesregierung unter Angela Merkel ist eine deutlich US-freundlichere, eine Russland-kritischere und eine besser europäisch integrierte, weniger (mittel-)machtorientierte deutsche Außenpolitik ${ }^{32}$ erwartet worden. Auch für diesen Bereich war bereits erwähnt worden, dass schon lange vor den Wahlen eine konvergente Entwicklung zwischen der Schröder-Regierung und der Merkel-Opposition eingesetzt hatte. Der Punkt der größten Distanz war vor Ausbruch des Irak-Krieges erreicht worden, schon bald danach bewegten sich die beiden Antagonisten wieder aufeinander zu, jedenfalls hinsichtlich ihres Verhältnisses zu den USA. Damit soll nicht geleugnet werden, dass ein gewisser Abstand zwischen den Positionen weiter bestand und die Erwartung rechtfertigte, dass Angela Merkel das Verhältnis zu den USA in die Tradition früherer deutscher Regierungen einfügen werde, das heißt in NATO und deutsch-amerikanischen Beziehungen ein Gleichgewicht - jedenfalls auf außen-, verteidigungs- und sicherheitspolitischem Gebiet - zur europäischen Integration und zur engen Freundschaft mit Frankreich wiederherstellen werde. In der Tat scheint diese Absicht im Koalitionsvertrag verankert zu sein, auch wenn hier schwer zu unterscheiden ist zwischen diplomatischen Bekenntnissen hohen Allgemeinheitsgrades und praktischen politischen Akzenten. Der Abschnitt des Koalitionsvertrages, der unter dem Titel ,Transatlantische Zusammenarbeit und europäische Sicherheitspolitik * steht, zielt auf dieses Gleichgewicht, es ist davon die Rede, dass beide zueinander ,nicht in Konkurrenz“ stehen, dass Europa „nicht als Gegengewicht, sondern als Partner“ der USA aufzufassen sei, dass sich ,europäische Außenpolitik und atlantische Partnerschaft im gemeinsamen Handeln bewähren“ müssen. ${ }^{33}$ Möglicherweise sind die inzwischen erkennbaren Aktzentsetzungen und Herausforderungen aussagekräftiger als der Koalitionsvertrag selbst: die Bestellung eines herausragenden Mitgestalters der Europäischen Sicherheitsstrategie ins Bundeskanzleramt, das Bemühen Angela Merkels, die NATO in Richtung eines Forums politischer Partnerschaft zu schieben (und damit vom militärischen Auftrag zu entlasten?) sowie vor allem die Auseinandersetzung um CIA-Flüge und -Verhaftungen über und in Deutschland - darauf wird zurückzukommen sein.

\section{Vom Koalitionsvertrag zur Regierungsbildung - Strukturen der deutschen Europapolitik}

Auf die Frage: ,Wer wird in der künftigen Bundesregierung die Europapolitik verantworten?“, antwortete Angela Merkel: „Die Europapolitik verantwortet immer der Kanzler beziehungsweise die Kanzlerin und alle, die in den Ressorts Verantwortung tragen." ${ }^{4}$ Diese diplomatische Formel lässt alle Bedeutung in dem unscheinbaren „und“ verschwinden, denn

31 Koalitionsvertrag, S. 128.

32 Vgl. Anmerkungen 3 bis 5 .

33 Koalitionsvertrag, S. 131.

34 Angela Merkel, Interview im Handelsblatt, 27.10.2005. 
gerade auf das Verhältnis zwischen Kanzleramt und den Ressorts kommt alles an. Dass es keine radikale Wende zu Gunsten einer Zentralisierung bei der Bundeskanzlerin, unter dem Hut eines Europaministers, geben würde - im Unterschied zu früheren Plänen -, war schon vor der Wahl klar. Eine Europa-Abteilung allerdings soll im Kanzleramt erhalten bleiben, sicher wiederum betraut vor allem mit den Vorbereitungen des Europäischen Rates, das heißt mit den Auftritten der Kanzlerin selbst auf europäischer Bühne. Freilich lassen Strukturen auch immer Raum für die Entfaltung von Persönlichkeiten: Reinhard Silberberg, bisheriger Leiter der Europaabteilung im Kanzleramt und hier Bürge dafür, dass der Output der relativ kleinen Abteilung weit über ihrer quantitativen personellen Ausstattung lag, wechselt als Staatssekretär ins Auswärtige Amt; sein Nachfolger wird Uwe Corsepius. Dagegen wird neben der Europaabteilung Christoph Heusgen als außen- und sicherheitspolitischer Berater der Bundeskanzlerin fungieren - und sicher aus seiner bisherigen Leitungstätigkeit im Politischen Stab von Javier Solana besonderes Interesse an der Außen-, Sicherheits- und Verteidigungspolitik, und hier wieder vor allem im Rahmen der Europäischen Union - mitbringen. Möglicherweise wird sich dementsprechend eine Aufgaben- und Arbeitsteilung einstellen, bei der sich die Europaabteilung im Bundeskanzleramt auf die Themenbereiche der wirtschaftlichen Integration konzentriert. ${ }^{35}$

Im Auswärtigen Amt herrscht ebenfalls eher Bedarf an Arbeitsteilung denn an Konzentration. Mit Reinhard Silberberg, dem bisherigen europapolitischen Sprecher der SPD-Fraktion Günter Gloser und Gernot Erler, ebenfalls SPD-Politiker, sind gleich drei Staatsminister beziehungsweise -sekretäre (trotz der ihnen zugewiesenen unterschiedlichen Schwerpunkte) auch mit Europa befasst. Wie zwischen ihnen, ihrem Minister, der Europaabteilung des Auswärtigen Amtes (weiterhin unter der Leitung von Eckart Cuntz) die europapolitische Koordinierung aufgeteilt, abgestimmt und organisiert wird, bleibt abzuwarten.

Nicht weniger kompliziert gestalten sich die Verhältnisse in anderen Ministerien. Die ehemalige Europaabteilung des Bundeswirtschaftsministeriums scheint ein Nomadenschicksal zu haben: Nach ihrer Abwanderung ins Bundesfinanzministerium vor sieben Jahren, auf Initiative von Oskar Lafontaine, tritt nun wenigstens ein Großteil, inklusive der Koordinierungsfunktion, den Weg zurück ins Wirtschaftsministerium an, so gewünscht von und zugestanden an Edmund Stoiber, eine Abmachung, die auch nach seinem Rückzug und seiner Ersetzung durch Michael Glos beibehalten wurde. ${ }^{36}$ Während im Bundeswirtschaftsministerium der dort neue Staatssekretär und ehemalige bayerische Europaabgeordnete Joachim Würmeling die Europa-Abteilung aufzubauen hat, verbleiben im Bundesfinanzministerium unter dem bisherigen Leiter Carsten Pillath diejenigen Teile der Abteilung, die sich mit Fragen der Geld- und Finanzpolitik sowie insbesondere mit der Währungsunion im engeren fachlichen Sinne befassen. Damit wird eine wichtige europapolitische Schaltstelle, die in der Vergangenheit - neben dem Bundeskanzleramt und dem Auswärtigen Amt - auch Koordinierungsfunktionen ausgeübt hat, dem Nachfolger von Hans Eichel, Peer Steinbrück, teilweise entzogen und in ein CSU-geführtes Ministerium eingegliedert.

Natürlich verzichten auch andere Ministerien, die von europäischen Politiken betroffen sind, nicht auf ihre Europa-Abteilungen. Darunter könnte die des Innenministeriums angesichts der stark gewachsenen Aufgaben gemeinsamer innerer Sicherheitspolitik an Bedeutung gewinnen, zumal auch sie unter die Federführung eines erfahrenen ,Europäers' in der Bundespolitik

35 Vgl. zum Personaltableau und seiner politischen Bedeutung auch Sabine Herre: Achse Tallinn-Berlin-Madrid, in: die tageszeitung, 23.11.2005, vgl. http://www.taz.de/pt/2005/11/23/a0145.nf/textdruck (letzter Zugriff: 05.12.2005).

36 Die genaue Regelung findet sich im Organisationserlass der Bundeskanzlerin vom 22. November 2005, Bundesgesetzblatt Teil I, Nr. 71, 25. November 2005, S. $3197 f$. 
gestellt wird: Der Saarländer Peter Altmaier, mit eigener Erfahrung als Kommissionsbeamter und langjähriger Bundestagsabgeordneter, aktiv im Europa-Ausschuss, übernimmt hier den Staatssekretärsposten, unter einem Minister Schäuble, dessen europapolitisches Interesse man denke zurück an die beiden ,Schäuble-Lamers Papiere‘ von 1994 und 1999 (und viele andere Stellungnahmen und Funktionen) - ihn auch als Innenminister nicht verlassen wird.

Das gesamte Tableau der Verteilung europapolitischer Aufgaben in der Bundesregierung wird damit eher noch bunter als bisher, von Zusammenfassung und Konzentration kann keine Rede sein. ${ }^{37}$ Die Gewichte sind auf komplizierte Weise zwischen dem CDU-geführten Bundeskanzleramt (Kanzlerin Merkel mit Heusgen und Uwe Corsepius), dem SPD-geführten Auswärtigen Amt (Minister Steinmeier mit Gloser, Silberberg und Erler als Staatsminister beziehungsweise -sekretäre und mit Cuntz als Abteilungsleiter), dem CSU-geführten Wirtschaftsministerium (Minister Glos mit Würmeling als Staatssekretär und einer neu aufzubauenden, koordinierenden Europaabteilung) aufgeteilt, es folgen das SPD-geführte Finanzministerium (Minister Steinbrück als SPD-Minister, Europaabteilung unter Leitung Pillath) und das wiederum CDU-geführte Innenministerium (Minister Schäuble mit Altmaier als Staatssekretär). Koordination bleibt damit sicher die Hauptaufgabe im ,shaping' der deutschen Europapolitik, aber ob sich damit das Auswärtige Amt noch deutlicher als Koordinator profilieren kann oder ob im Bundeskanzleramt das Gravitationszentrum entsteht muss vorerst dahingestellt bleiben.

Jedenfalls erscheint es verfrüht, aus der personellen und strukturellen Konstellation zukünftige Konflikte zwischen der angeblichen Fortsetzung einer Schröderschen Außenpolitik einerseits und einer christdemokratisch-Merkelschen andererseits abzuleiten ${ }^{38}$ - einige Kommentatoren haben den Versuch unternommen, hier Konfliktpotenzial mit Sprengkraft für die Große Koalition insgesamt aufzufinden. ${ }^{39}$ Anlass war unter anderem ein Interview von Christoph Heusgen, der das deutsche Streben nach einem UNO-Sitz als eine „Illusion“ bezeichnet hatte und von Gernot Erler dafür gerügt wurde, dass er den Koalitionsvertrag nicht ernst nehme ${ }^{40}$ - dort heißt es allerdings sibyllinisch: „Deutschland bleibt bereit, auch mit der Übernahme eines ständigen Sicherheitsratssitzes mehr Verantwortung zu übernehmen. In der Perspektive streben wir weiterhin einen ständigen Sitz für die EU an. ${ }^{\text {“41 }}$ Diese Kontroverse hat aber kaum für Konfliktstoff über den zeitlichen Horizont journalistischen Interesses hinaus gesorgt, und selbst in den meisten Pressebeiträgen dominiert die Annahme von Kontinuität in der deutschen Europa- und Außenpolitik - trotz der personellen Neubesetzungen und strukturellen Nuancen zwischen Kanzleramt und Ministerien, vielleicht aber sogar wegen der noch gewachsenen Systematik von ,Checks and Balances', von vielfältigen Akteuren $^{42}$, die zu koordinieren immer mehr auch diplomatisches Geschick verlangen wird,

37 Auch in dieser Hinsicht trägt die neue Lage der Hoffnung Jannings auf eine ,wirksame Bündelung des Regierungshandelns" nicht Rechnung, Janning: Bundesrepublik Deutschland, hier S. 316.

38 Diese Ansicht teilt Sabine Herre: „Achse Tallinn-Berlin-Madrid“, in: die tageszeitung, 23.11.2005. Ähnlich Jan Ross: „Das Vorstellungsgespräch“, in: Die Zeit, 49/2005, S. 4.

39 So Stefan Kornelius: „Außen vor“, in: Süddeutsche Zeitung, 24.11.2005, S. 4: „Vermutlich gibt es kein anderes Fach der neuen Regierungspolitik, wo das Konfliktpotenzial so offensiv verschwiegen wurde." Vgl. dagegen die entgegengesetzte These von Andreas Rinke: „Eine Köchin, drei Köche“, in: Handelsblatt, 20.10.2005.

40 So auch Herre: „Achse Tallinn-Berlin-Madrid“.

41 Koalitionsvertrag, S. 136.

42 So auch Andreas Rinke, Handelsblatt 20.10.2005: „Gegen einen radikalen Bruch spricht schon, dass in Berlin künftig eine Köchin und gleich drei Nebenköche die deutsche Europapolitik bestimmen werden. Nach einem Fortschritt gegenüber der Konstellation ,Koch und Kellner' unter Kanzler Schröder und Außenminister Joschka Fischer sieht dies erst einmal nicht aus. Stattdessen werden die von der SPD geführten Außen- und Finanzressorts eifersüchtig über ihre Zuständigkeiten wachen." 
eine Überlegung, die einerseits für eine starke Rolle des Auswärtigen Amtes im Zusammenspiel mit dem Bundeskanzleramt spricht, andererseits auch letzterem selbst eine stärker ausgeprägte Schiedsrichterrolle zuweisen könnte. Nicht zuletzt wird die Rolle des deutschen Botschafters im Ausschuss der Ständigen Vertreter entscheidend sein, auch wenn sie weisungsgebunden ist - mit Wilhelm Schönfelders Verbleib an dieser wichtigen Schaltstelle zwischen deutschen und europäischen Institutionen ist auch hier für Kompetenz und Kontinuität gesorgt.

Damit nicht genug: Auch der Bundestag, auch die Bundesländer nehmen Einfluss auf die deutsche Europapolitik, ihre strukturellen Positionen im Gefüge der Akteure gilt es zu beachten. Die Bundesländer haben in zwei Schritten ihre Stellung befestigt, ja gestärkt, zum einen bei der Abstimmung über den Europäischen Verfassungsvertrag, zum anderen im Rahmen der Einigung, die die Föderalismus-Reform-Kommission während der Koalitionsverhandlungen erzielt hat.

Bereits im Mai hatten die Bundesländer durchsetzen können, dass ihre europapolitische Rolle, wenn auch nur um Nuancen, gestärkt wird - im Gegenzug waren sie bereit, noch vor dem französischen Referendum dem Europäischen Verfassungsvertrag zuzustimmen und so ein (freilich seine Wirkung verfehlendes) Signal nach Frankreich zu senden. Das Ergebnis dieses Interessenausgleichs zwischen Bund und Ländern ist ein Gesetz, das zwar erst mit InKraft-Treten des Europäischen Verfassungsvertrages seinerseits in Deutschland in Kraft treten soll, angesichts der unsicheren Perspektive der Verfassung nun schon früher wirksam werden könnte. Dabei geht es insbesondere um die Umsetzung des so genannten Frühwarnsystems, das der VVE vorsieht und demzufolge die nationalen Parlamente früher als bisher in den Gesetzgebungsprozess der Union eingeschaltet werden sollen, so früh, dass sie gegebenenfalls Verstöße gegen das Subsidiaritätsprinzip vor dem Abschluss des Gesetzgebungsverfahrens aufhalten können. Freilich lässt sich das vom VVE vorgesehene Klagerecht vor dem Europäischen Gerichtshof nicht vor In-Kraft-Treten der Verfassung umsetzen, wohl aber die Einbeziehung der nationalen Parlamente - und das heißt in Deutschland eben beider Kammern, auch des Bundesrates und damit der Länder. Das schon verabschiedete Gesetz „,̈̈ber die Ausweitung und Stärkung der Rechte des Bundestages und des Bundesrates in Angelegenheiten der Europäischen Union" 43 sollte zeitgleich mit dem VVE in Kraft treten, könnte aber jedenfalls hinsichtlich der Teile, die nicht vom VVE abhängig sind, schon bald angewandt werden ${ }^{44}$.

Der zweite Schritt, mehr zur Präzisierung als zur Stärkung der Länderbeteiligung an der Europapolitik, wurde in der Föderalismus-Kommission getan und hat Eingang in den Koalitionsvertrag gefunden. Demzufolge soll der Absatz 6 des Art. 23 GG geändert werden, ganz auf der Linie der Föderalismus-Reform im Allgemeinen: Die Länderkompetenzen sollen enger begrenzt, dafür die Länder aber mit eindeutigerer Autonomie ausgestattet werden. Statt der Formulierung, dass Ländervertreter dann im Ministerrat Rechte wahrnehmen ,sollen“, wenn ,im Schwerpunkt ausschließliche Gesetzgebungsbefugnisse der Länder betroffen sind“, heißt es nun präzise, dass ,auf den Gebieten der schulischen Bildung, der Kultur oder des Rundfunks“ die Wahrnehmung der Rechte den Ländern übertragen „wird““ ${ }^{5}$

43 Bundesgesetzblatt 2005, Teil I, Nr. 71, S. 3178, 17. November 2005, http://217.160.60.235/BGBL/bgbl1f/ bgbl105s3178.pdf (letzter Zugriff: 16.12.2005).

44 Vgl. dazu auch allgemeiner Daniela Kietz: Der Bundestag in der Europapolitik. Stiftung Wissenschaft und Politik, Mai 2005 (SWP-Aktuell 19), http://www.swp-berlin.org/common/get_document.php?id=1265 (letzter Zugriff: 16.12.2005).

45 Koalitionsvertrag, Anhang: Ergebnis der Koalitionsarbeitsgruppe zur Föderalismus-Reform, S. 28: IV: Europatauglichkeit. 
Die letztgenannte Komponente ist wohl die einzige, die die Strukturen deutscher Europapolitik vereinfacht. Alle anderen genannten Arrangements struktureller, personeller und institutioneller Natur bestätigen oder komplizieren gar noch weiter den komplizierten politischen Prozess auf dem Weg zu einer einheitlichen, kohärenten, abgestimmten und möglichst früh erkenn- und handhabbaren deutschen Position.

\section{Erste Schritte der neuen Regierung - Reisesymbolik}

In einem gewissen Widerspruch zu der geringen Bedeutung europäischer Themen im Wahlkampf ist die erste politische Aktivität, die die neue Bundesregierung nach ihrer Wahl am 22. November 2005 entfaltet hat, europäischer Natur gewesen: Die ersten Tage im Amt haben die neue Bundeskanzlerin und ihr Außenminister auf Reisen in Europa verbracht. Dabei lag die Aktivität selbst wesentlich im Reisen an sich, nicht so sehr in der Lösung substanzieller Probleme europäischer Politik: Die Reihenfolge der Besuche hatte symbolische Bedeutung, und der Stil der Begegnungen ist vielfachen Interpretationen ausgesetzt gewesen.

\section{Paris}

Die erste Reise führte Angela Merkel und Frank-Walter Steinmeier am Tag nach der Wahl der Regierung, am 23. November, gemeinsam nach Frankreich, zu Jacques Chirac, der beide mit ausgesuchter, seinerseits zu weitreichender Deutung Anlass gebender Höflichkeit empfing. Die Geste dieses Besuches und die Gesten während des Aufenthaltes in Paris bedeuteten europapolitische Kontinuität (einmal mehr) hinsichtlich der herausragenden Rolle, die das Verhältnis zu Frankreich für die deutsche Politik spielt. ${ }^{46}$ Obwohl ein solcher Antrittsbesuch in Frankreich inzwischen eine Art Gepflogenheit für gerade neu gewählte deutsche Bundeskanzler ist, waren doch der überaus prompte Antritt der Reise und der besonders zuvorkommende Empfang nicht allein Routine, umso weniger als während des Bundestagswahlkampfes sowohl der französische Staatspräsident als auch der Premierminister Dominique de Villepin keinen Hehl aus ihrer Sympathie für einen Wahlsieg Gerhard Schröders gemacht hatten, der eine so enge Anbindung an Frankreich, an Chirac zumal, gefunden hatte, dass jede Alternative für die französischen Staatslenker nur schlechter scheinen konnte. Zudem war auch noch vorausgesagt worden, dass Angela Merkel das Verhältnis zu Frankreich lockern und dagegen eine offenere Partnerschaft, mit anderen, ausgleichenden Gewichten, etwa Großbritannien und den kleineren wie östlicheren EU-Partnern, anstreben werde. Darüber hinaus fürchtete man in Frankreich, sie werde auch eine US-freundlichere Politik betreiben und Frankreich damit wieder mit seiner Resistenz gegen Amerika allein lassen. Nicolas Sarkozy, Innenminister und chancenreicher Kandidat für die Präsidentschaftswahlen 2007, gehörte zu den wenigen, die von Anfang an auf Angela Merkel gesetzt hatten. Die Reise nach Paris war unter diesen Umständen mehr als eine freundliche RoutineÜbung, sie bestätigte den herausragenden Rang Frankreichs als Partner Deutschlands gegen Zweifel, unabhängig von konkreten Einzelinteressen - solche scheinen bei dieser Gelegenheit nicht zur Sprache gekommen zu sein oder waren schon zuvor durch Festlegungen im Koalitionsvertrag harmonisiert worden, wie vor allem der Agrarkompromiss des Jahres 2002.

46 Dies gilt nicht nur für die Politik, sondern auch für das Bewusstsein der Öffentlichkeit: Auf die Frage nach dem engsten Verbündeten antworten die befragten Deutschen mit deutlicher relativer Mehrheit (33\%) „Frankreich“, gefolgt von den USA (28\%); vgl. Ralf Beste/Georg Mascolo/Ralf Neukirch: „Kalter Frieden“, in: Der Spiegel, 48/2005, 28.11.05, S. 22-25. 


\section{Brüssel}

Die zweite Etappe derselben Reise gab nicht weniger Anlass zu Deutungen, sie führte Kanzlerin und Außenminister, immer noch einträchtig auf gemeinsamem Antrittsbesuch gedeutet als Zeichen großkoalitionärer Übereinstimmung -, nach Brüssel und dort gleich zu drei Besuchsgesten, zuerst zur NATO (zu ihrem Generalsekretär), dann zur Europäischen Union (zum Kommissions- und Parlamentspräsidenten) und schließlich zum belgischen Regierungschef. Es geht wohl zu weit, auch noch in der Reihenfolge der Brüsseler Agenda erst NATO, dann Europäische Union - Bedeutung zu suchen, aber soviel ist gewiss, dass gleich nach dem wichtigsten bilateralen Freund und Partner Frankreich die beiden inter- beziehungsweise supranationalen Organisationen standen, dass mithin die Außenpolitik Deutschlands europäisch und transatlantisch sein will, multi- und supranational mehr als zwischenstaatlich. Auch dieses Signal ist bedeutsam, und kann auf einen Unterschied zu Schröders mehr und mehr an nationalstaatlichen Eigeninteressen orientierter Außenpolitik deuten: Keine Rede mehr von ,Mittelmacht" oder ,Zentralmacht', sondern Wiedereingliederung in den Mainstream europäischer Integration und transatlantischer Partnerschaft, und zwar mit gleichem Nachdruck auf beiden - so lässt sich die Zeichensprache der Reisesymbolik deuten.

Kaum zur Geltung kam in der Öffentlichkeit eine weitere nicht unwichtige Nuance dieser Inszenierung von politischen Gesten, die doch gerade auf der erwarteten Linie liegt, nämlich das Zusammentreffen Angela Merkels auch mit dem belgischen Premierminister Guy Verhofstadt - und schon am Vorabend ihrer Wahl zur Bundeskanzlerin hatte sie in Berlin den luxemburgischen Premier Jean-Claude Juncker empfangen: Kleinere EU-Partner zählen auch, so die Botschaft, eine Stilfrage und doch mehr als das, zwar kein Kontinuitätsbruch mit der deutschen Europapolitik, aber sehr wohl eine Änderung in der Rolle und Haltung Deutschlands im Kreis der EU-Partner gegenüber Schröders Neigung, sich um das Gefühl der ,Kleinen“, als quantité négligeable behandelt zu werden, keine Sorgen zu machen.

\section{London}

Erst nach Brüssel trennten sich die Wege von Kanzlerin und Außenminister, letzterer reiste weiter in die Niederlande und rundete damit die Benelux-Besuche ab, Angela Merkel flog - erst jetzt oder schon jetzt? - nach London, wo man sich ebenfalls Sorgen um und Hoffnungen auf eine wieder engere, weil vielleicht liberalere und amerikafreundlichere deutsche Partnerin machte. Vielleicht war dieser Besuch am meisten auf die bloße Geste der Höflichkeit gegenüber dem nächstgrößeren EU-Partner zu reduzieren, denn Substanz für die heimliche Hoffnung Tony Blairs und seiner Regierung wurde nicht geliefert. ${ }^{47}$

\section{Warschau}

Von größerer Signifikanz war der nächste europäische Besuch der Kanzlerin, auch wenn einige Tage vergingen, bis es dazu kam: Polen war das nächste Ziel, und hier galt es nicht nur, charmante Gesten auszutauschen, sondern handfeste aktuelle Verstimmungen zu dämpfen oder auszuräumen, und dies im Kontakt mit einer neuen, rechtskonservativen Regierung,

47 Jannings Hoffnung auf ein deutsch-französisch-britisches Trio, das nicht nur Blockade-, sondern auch Gestaltungsmacht aufbringen würde, scheint nicht in Erfüllung zu gehen, Janning: Bundesrepublik Deutschland. Auch die Einigung über die mittelfristige Finanzplanung, obwohl ihr Kernstück die Vermittlung zwischen französischen und britischen Interessen durch Angela Merkel ist, deutet nicht auf ein Dreigestirn hin, sondern lässt eher die traditionellen Konturen eines deutsch-französischen Tandems plus rücksichtsvoller Einbeziehung kleinerer (oder der ,Großen unter den Kleinen“, wie Polen) erkennen. 
die nicht zuletzt durch ihren europa- und deutschlandkritischen Wahlkampf an die Macht gekommen war. Insbesondere das in Berlin geplante ,Zentrum gegen Vertreibungen " erweckte auf polnischer Seite Befürchtungen, vorgetragen vor allem vom neuen polnischen Präsidenten Lech Kaczynski. Die Formel im Koalitionsvertrag, der zufolge „ein sichtbares Zeichen“ der Erinnerung an das „Unrecht der Vertreibungen“ (immer auch wird nicht nur von der Vertreibung der Deutschen, sondern von „Vertreibungen“ im Plural gesprochen) gesetzt werden solle, ließ Angela Merkel immerhin Spielraum für Entgegenkommen in Warschau. Der zweite Stein des Anstoßes, der für die Bundeskanzlerin in Warschau lag, betraf die zwischen ihrem Vorgänger und seinem Freund Putin geplante Ostsee-Gaspipeline, die zum Leidwesen Polens (und der baltischen Staaten) eine Ausklammerung der polnischen (und baltischen) Interessen bedeutete, zu Gunsten einer deutsch-russischen Interessengemeinschaft - in polnischen Zeitungen wurde der Begriff des „Schröder-Putin-Paktes“ bemüht. ${ }^{48}$ Auch hier konnte die Kanzlerin auf polnische Ängste eingehen, indem sie eine gemeinsame Arbeitsgruppe zur weiteren Entwicklung des Projekts sowie möglicherweise eine Stichpipeline nach Polen in Aussicht stellte - Nuancen der Einlösung des angekündigten Versprechens, mehr als Gerhard Schröder auf Vermittlung mit kleineren und insbesondere ostmitteleuropäischen EU-Partnern zu setzen.

\section{Erste europapolitische Aufgaben und Herausforderungen}

Die Polen-Reise befand sich bereits im Übergang von den symbolischen Eröffnungsgesten der neuen Bundesregierung hin zu konkreten Aufgaben, die sich bei den folgenden Treffen und Besuchen - bei Jacques Chiracs Gegenbesuch in Berlin am 8. Dezember etwa - immer mehr in den Vordergrund schoben und schon vier Wochen nach dem Regierungsantritt einen Gipfel erreichten, bei dem Erfolg oder Misserfolg der britischen Ratspräsidentschaft, und weit mehr als das, nämlich Einigung oder Scheitern in der Auseinandersetzung um die mittelfristige Finanzplanung der Europäischen Union auf dem Spiel standen. Damit war ein fliegender Wechsel von Symbolik zu handfesten Verhandlungen innerhalb weniger Tage vorgegeben, der die neue Kanzlerin - vor allem sie, denn ihr neuer Außenminister Steinmeier hatte gleichzeitig mit der Problematik von CIA-Flügen und möglicherweise sogar Entführungen in, aus und über Deutschland zu kämpfen - mehr noch als die vorangegangenen Besuche als Vermittlerin forderte. In der Tat lagen die Positionen im Finanzstreit so weit auseinander, dass es viel zu vermitteln gab. ${ }^{49}$

Nach Vorschlägen der Kommission und des Europäischen Parlaments hatte der luxemburgische Premierminister Jean-Claude Juncker bereits im Juni eine Einigung versucht, in der alle Parameter berücksichtigt waren, die auch jetzt miteinander in Einklang zu bringen waren, an erster Stelle die Opposition zwischen dem britischen Insistieren auf dem Rabatt und dem französischen Beharren auf der finanziellen Ausstattung der Agrarpolitik. Daneben gab es aber auch das Gegenüber zwischen den Nettozahlern auf der einen und (vor allem) den neuen EU-Mitgliedstaaten auf der anderen Seite - während die ersteren an einem Haushaltsvolumen von nicht (viel) mehr als ein Prozent des EU-Bruttonationaleinkommens festhalten wollten, waren die letzteren an einer Erhöhung zur Förderung ihrer nachholenden

48 So das polnische Magazin Wrpost, zitiert nach: Daniel Wortmann: Deutsch-polnische Beziehungen auf dem Prüfstand, vgl. http://www.dw-world.de/dw/article/0,2144,1798889,00.html (letzter Zugriff: 05.12.2005); zum Hintergrund vgl. Roland Götz: Die Ostseegaspipeline. Instrument der Versorgungssicherheit oder politisches Druckmittel?, Stiftung Wissenschaft und Politik, September 2005 (swp-aktuell 2005/41), http://swp-berlin.org/ common/get_document.php?id=1391 (letzter Zugriff: 16.12.2005).

49 Allerdings ist hier nicht der Raum, die ganze Problematik der Finanziellen Vorausschau auszubreiten. 
Entwicklung interessiert. Beide Konflikte waren miteinander verknüpft, insofern eine Reduktion des britischen Rabatts bedeutet, dass Großbritannien auch stärker an den Erweiterungskosten beteiligt wird und zudem die Belastung für die Nettozahler sich verringern würde - Gleiches gilt auch für eine Infragestellung (und als Folge davon der Verringerung) der Kosten für die Agrarpolitik. Schließlich hing eine Einigung über die Verteilung von Lasten und Nutzen natürlich auch vom Gesamtvolumen des Pakets ab.

Offensichtlich war, dass die britischen Vorschläge - in ihrer ursprünglichen wie in ihrer revidierten Fassung - nicht konsensfähig waren und auch keine Kompromisslinie erkennen ließen ${ }^{50}$. Ein Scheitern auch des - nach dem luxemburgischen - zweiten Versuchs, sich auf die Finanzielle Vorausschau zu einigen, war demnach durchaus nicht unwahrscheinlich. Dann hätte freilich die Last der Einigung auch noch die deutsche Präsidentschaft im ersten Halbjahr 2007 ereilen können - die britische Präsidentschaft hatte verlauten lassen, dass es 2006 keine Einigung geben werde, wenn jetzt keine erzielt würde -, und die Finanzierung der Europäischen Union am Anfang der Periode 2007-2013 in schwere Turbulenzen gebracht.

Noch am 15. Dezember schätzten Beobachter „Merkels Ausflug ins blockierte Europa“ als nahezu chancenlos ein: Eine „Einigung über den EU-Haushalt ist in weiter Ferne“, ,mit Gerhard Schröder fehlt ein erfahrener Vermittler. Merkel versucht sich zwar in der Rolle, weiß aber selbst, dass sie noch nicht für voll genommen wird. ,Nach drei Wochen im Amt wäre das Hybris', heißt es in der Regierung. "51 Die Spannung vor dem Gipfel war jedenfalls übergroß, die Erwartungen an die deutsche Kanzlerin dagegen sehr gedämpft - die Überraschung und Anerkennung für den Erfolg im gleichen Maße unerwartet. Denn nur zwei Tage später, nach dem Gipfel, am 17.12., titelte die Süddeutsche Zeitung bereits „,Der MerkelGipfel“52, und am Tag darauf schwang sich die FAZ zu dem Titel „Angela, der Gipfel-Engel" ${ }^{\prime 53}$ auf, ein Enthusiasmus, den die Bild am Sonntag teilte, indem sie auf der ersten Seite von „Merkels Triumph-Nacht" ${ }^{“ 54}$ sprach.

Der Erfolg hatte damit begonnen, dass Angela Merkel und Frank-Walter Steinmeier die britischen Vorschläge nicht rundweg als inakzeptabel bezeichnet, sondern Verhandlungsbereitschaft signalisiert und dafür geworben hatten. Der zweite entscheidende Schritt war die Vorlage eines mit Frankreich abgestimmten Kompromissvorschlags, der den französischen Partnern die Sicherheit gab, dass das übliche Tandem funktionierte und Deutschland nicht irgendwo zwischen Frankreich und Großbritannien seinen Platz sucht. Dieser Vorschlag lag ziemlich genau auf der Hälfte der von der britischen Präsidentschaft zuletzt angebotenen Gesamtsumme von $849 \mathrm{Mrd}$. Euro und dem luxemburgischen Vorschlag von $872 \mathrm{Mrd}$. Euro, nämlich bei $862 \mathrm{Mrd}$. Euro - auf diese Summe hat sich der Gipfel dann schließlich in der Tat geeinigt. Drittens ist si-

50 Trotz einer britischen Strategie, den eigenen Rabatt durch individuelle ,Geschenke ‘ an einzelne Partner zu retten, so etwa durch Entgegenkommen an Deutschland bei der Nettozahlerposition oder bei Ausnahmeregelungen in Sachen Dienstleistungsrichtlinie - eine Strategie, der Elmar Brok ,teuflische Genialität“ bescheinigte, die aber letztlich nicht zu mehr Zustimmungsbereitschaft, sondern zu genereller Empörung führte, zitiert nach Cornelia Bolesch: „Spalter Blair. Neue EU-Mitglieder werfen London brutale Politik vor“, in: Süddeutsche Zeitung, 15.12.2005, S. 6.

51 Carsten Volkery: „Merkels Ausflug ins blockierte Europa“, Spiegel online Politik, 15.12.2005; http:// www.spiegel.de/politik/ausland/0,1518,390540,00.html (letzter Zugriff: 18.12.2005). Die skeptische Erwartungshaltung wurde von vielen Beobachtern geteilt.

52 Süddeutsche Zeitung, 18.12.2005; http://www.sueddeutsche.de/,tt3m3/ausland/artikel/398/66332/ (letzter Zugriff: 18.12.2005).

53 FAZ.NET, http://www.faz.net/s/Rub99C3EECA60D84C08AD6B3E60C4EA807F/Doc EA471A20AD84C 469A9A38DC5F17CC8C2E ATpl Ecommon Scontent.html (letzter Zugriff: 18.12.2005).

54 Bild am Sonntag, 18.12.2005. 
cher die hochprofessionelle und effiziente Vorbereitung durch den Außenminister und seine Mitarbeiter im Auswärtigen Dienst, das heißt vor allem in Brüssel selbst, eine Bedingung für den Erfolg gewesen. Allen Beobachtern zufolge sei aber Angela Merkels Einsatz während des Gipfels selbst, und insbesondere in seiner kritischen, entscheidenden Phase am Mittag und Nachmittag des Freitags (16.12.2005) den Ausschlag gegeben. Dabei soll sowohl ihre Bereitschaft, unermüdlich in den unterschiedlichsten Gesprächskonstellationen für einen Kompromiss zu werben als auch ihre Fähigkeit, die finanziellen und politischen Implikationen der jeweiligen Vorschläge schnell richtig einzuschätzen, von besonderer Bedeutung gewesen sein, wie letztlich auch ihre von allen Seiten anerkannte Vertrauenswürdigkeit. Es scheint auch Angela Merkel gewesen zu sein, die zunächst eine prinzipielle Einigkeit über die Gesamtsumme herbeigeführt hat, so dass dann, am weiteren Freitag Abend und in der Nacht, über die Details der Aufteilung dieser Summe gefeilscht werden konnte - auch wenn diese Verhandlungen nicht mehr die gleiche Gefahr für den Erfolg bargen wie die Frage der Gesamtsumme, überaus zäh und langwierig waren sie dennoch. Noch einmal und auch in dieser Phase hat Angela Merkel in doppelter Weise einem Durchbruch zumindest den Weg geebnet, indem sie nämlich zunächst Tony Blair dazu bewegen konnte, erhebliche Abstriche an Großbritanniens Rabatt-Hoffnungen zu akzeptieren (und dafür ,nur' die prinzipielle Bereitschaft Frankreichs aufbot, die Finanzierung der Agrarpolitik 2008 im Rahmen einer allgemeinen ,Revision' des EU-Haushalts zu überprüfen $)^{55}$, und dann bereit war, 100 Millionen Euro aus ,deutschen Anteilen' an Polen zur Förderung ostpolnischer Regionen abzutreten. Das machte den polnischen Ministerpräsidenten nach eigener Aussage regelrecht glücklich ${ }^{56}$, und wird sicher mit der Hoffnung auf eine dauerhafte Auswirkung auf die deutsch-polnischen Beziehungen zugestanden worden sein. ${ }^{57}$

Mit alledem sind in der Tat die Hoffnungen auf einen ,Stilwechsel ' der deutschen Europapolitik mehr als erfüllt worden, ja es hat sich geradezu eine paradigmatische Konfiguration der bestmöglichen deutschen Rolle herausgeschält, gekennzeichnet durch die folgenden fünf Komponenten: (1a) Auf der Grundlage eines in enger Kooperation mit Frankreich abgestimmten Vorschlages, (1b) der auf deutscher Initiative beruhte, wurde (2) eine Abstimmung mit Großbritannien gesucht und gefunden, (3a) zugleich aber der Eindruck eines „,directoire" vermieden ${ }^{58}$, sondern mit Sensibilität und Rücksicht auf kleinere EU-Partner gehandelt, (3b) unter denen Polen an erster Stelle stand. ${ }^{59}$

55 Vgl. den Beitrag von Kai Oppermann in diesem Heft.

56 Vgl. Wulf Schmiese: „Angela Merkel beeindruckt Europa“, in: Frankfurter Allgemeine Sonntagszeitung, 18.12.2005, S. 1: ,'Das war eine wunderbare, bewegende Geste', lobte der polnische Ministerpräsident Kazimierz Marcinkiewicz die Kanzlerin. ,Der Sieg schmeckt wie guter französischer Champagner.'“

57 Dagegen trug dieser Zug der Kanzlerin in Deutschland die Forderung nach Ausgleich von Seiten der ostdeutschen Bundesländer ein; vgl. Cornelia Bolesch/Jens Schneider: Milbradt klagt über Kürzung der EU-Hilfen, in: Süddeutsche Zeitung, 19.12.2005; ähnlich Thüringens Ministerpräsident Althaus: „Über Kompensation verhandeln“, in: Tagesspiegel, 19.12.2005.

58 Auch die Konzeption eines „Führungstrios“, die William Wallace noch vor kurzem diskutierte, wurde damit von Angela Merkel nicht bestätigt, vgl. William Wallace: Das Führungstrio Deutschland - Frankreich - Großbritannien: Europas zentrales Problem?, in: integration 4/05, S. 328-331.

59 Es würde hier zu weit führen, alle Fragen an den und nach dem Gipfel zu erörtern, wie etwa die, ob die EU-25 nun - entgegen vielen Erwartungen - ihre Handlungsfähigkeit auch unter den Bedingungen des Vertrages von Nizza bewiesen hat, ob und in welchem Maß Angela Merkels Rolle nur einmal so gespielt werden konnte, nämlich als die eines Neulings im Kreis der anderen Regierungschefs, ob es Grenzen des wünschbaren Erfolgs gibt, die man besser nicht überschreitet, um andere Mitgliedern des Europäischen Rates nicht zu deutlich in den Schatten zu stellen - alle diese Fragen sind in den Medien gestellt, wenn auch selten schlüssig beantwortet worden. $\mathrm{Zu}$ den Grundzügen und dem Verhaltensmuster der neuen Bundesregierung, vgl. auch Hans-Peter Schwarz: Das Ende der Übertreibungen, in: Internationale Politik 8/2005, S. 8-15, wo es heißt: „Das Geheimnis erfolgreicher deutscher Außenpolitik heißt Ausgleich, Mäßigung und Vermittlung,"S. 8. 


\section{Aussichten in weiterem Horizont}

Der erfolgreiche Gipfel mit der Einigung über die finanzielle Vorausschau öffnet Perspektiven auf die weitere europapolitische Dimension. Die deutsche Ratspräsidentschaft 2007 wird nun nicht mehr unter dem Zwang stehen, die Finanzen der kommenden Jahre in aller Eile doch noch zu ordnen, der Blick muss sich nicht mehr allein auf den Boden der kleinsten Tatsachen richten, sondern kann erneut die Höhe eines Leitbildes anvisieren.

In dieser Richtung werden drei Perspektiven erkennbar: Zum einen besteht selbst in dieser Kategorie zum Teil Kontinuität zwischen Gerhard Schröder und Angela Merkel in der Hinsicht, dass beide die Globalisierung als die große Herausforderung sehen - und Europa als wesentlichen Teil der Antwort. Schröder hat aber freilich daneben eine bilaterale Außenpolitik mit ausgewählten Partnern (Paris, Moskau) und individuellen deutschen Interessen im Zentrum (UNO-Sitz) betrieben - eine Strategie, die für die europäische Integration keine bedeutende, jedenfalls nicht die herausragende Leitbild-Rolle vorsah. Angela Merkel dagegen könnte darauf zurückkommen, dass angesichts der engen Grenzen nationalstaatlichen Einflusses auf globale Entwicklungen die Union zu einer wirklichen Gestaltungsmacht ausgebaut werden muss - im deutschen Interesse, das sich einmal mehr als deckungsgleich mit dem europäischen erweist. Es scheint, dass die Lissabon-Strategie ein politisches Projekt dieser Tragweite werden könnte, wenn sie denn in der zweiten Hälfte ihrer Laufzeit wirklich als zentrale politische Aufgabe begriffen wird. Dass Angela Merkel ,Lissabon' in vielen ihrer europapolitischen Stellungnahmen an erster, herausragender Stelle genannt hat, wurde schon bemerkt und könnte darauf hindeuten, dass sie hier den Schwerpunkt setzen will. Dafür sprechen auch manche Gemeinsamkeiten zwischen ,Lissabon" und ihrem eigenen politischen Credo: die Privilegierung von Wettbewerbsfähigkeit und Wirtschaftswachstum, mittelbar zwar auch mit sozialpolitischen Zielen, aber doch in der spezifischen Konstellation, dass Wettbewerb und Wachstum als Voraussetzungen und beste Mittel zugleich für die Sozialpolitik gesehen werden. Auch der SPD und insbesondere dem Außenminister müsste die Lissabon-Strategie liegen, da sie doch in hohem Maß Vernetzung zwischen unterschiedlichen, innovativen Politikbereichen als Voraussetzung für globale Wettbewerbsfähigkeit annimmt.

Problematisch bleibt die Frage nach der integrationspolitischen Natur der Lissabon-Strategie. Im Unterschied zu früheren mobilisierenden und die Integration vorantreibenden Projekten wie dem Binnenmarkt oder der Währungsunion (und, viel früher schon, der Kohle- und Stahlgemeinschaft) ist die Lissabon-Strategie im Wesentlichen eine Selbstverpflichtung der Mitgliedstaaten, bestimmte Politiken in eigener Regie zu realisieren - europäische Institutionen, europäische Rechtsetzung, europäische Solidarität, gemeinsames Handeln spielen dabei keine herausragende Rolle. Insofern mag die Lissabon-Strategie für die Bewältigung globalisierungsbedingter politischer Aufgaben der Mitgliedstaaten eine bedeutende Rolle spielen, als integrationspolitisches Leitbild, gar als ,Finalitäts'-Szenario taugt sie kaum, weil sie keine qualitative Fortentwicklung der Europäischen Union als politisches System, als polity, im Gefolge hat.

Immer wieder hat Angela Merkel aber auch die Erneuerung einer Sozialen Marktwirtschaft und ihre Durchsetzung auf europäischer Ebene zu einem vorrangigen Ziel erklärt ${ }^{60}$.

60 Welche Tiefendimensionen ein solches Projekt erreichen kann, auf welchen Grundlagen es ruhen würde, skizziert Bernhard Schulz: „Beruf als Berufung“, in: Der Tagesspiegel, 11.12.2005, wo die These entwickelt wird, „,mit Angela Merkel kehre ein protestantisch-nüchterner Politikertyp zurück, wie ihn vor 100 Jahren Max Weber beschrieb" - eine weit reichende Interpretation, die einerseits der Pragmatik Angela Merkels ein religiöses Fundament unterstellt, zugleich auch die tieferen Rechtfertigungsgründe für ein politisches Projekt wie die Soziale Marktwirtschaft auf europäischer Ebene liefert - zumal wenn man ,die protestantischen Wurzeln der Sozialen Marktwirtschaft" (so der Titel einer von Günter Brakelmann und Traugott Jähnichen im Auftrag der Evangelischen Kirche in Deutschland herausgegebenen Textsammlung, Gütersloh 1994) mit bedenkt. 
Ergibt sich aus dieser Zielsetzung ein polity-relevantes Leitbild für die Europäische Union? Einer der Gründe für das Scheitern des Verfassungsvertrages, zumindest im französischen Referendum, war die Kritik, die Europäische Union sei zu neoliberal, sei nicht sozial genug, es fehle ihr, kurz gesagt, der Ausgleich zwischen Markt- und Sozialpolitik, eben dieses Gleichgewicht, das für die Soziale Marktwirtschaft konstitutiv ist. Es kann hier dahingestellt bleiben, wie berechtigt diese Kritik gerade am Verfassungsvertrag ist, der doch eher auf einen Angleich dieser Gewichte hinarbeitet, jedenfalls im Vergleich zu den geltenden Verträgen, und gerade um der skizzierten Forderung willen hätte angenommen werden können. Aber die populäre Kritik an der angeblichen sozialpolitischen Kälte der Union legt immerhin den Finger auf ein Charakteristikum des Verfassungsvertrages, das ihn von früheren epochemachenden Verträgen unterscheidet: Er tritt nicht mit einem klar erkennbaren politischen Projekt hervor - einem Projekt, um dessentwillen die Europäische Union eine grundlegende Vertragsänderung bräuchte. Demokratisierung, Effizienzsteigerung und Werteorientierung, als polity-orientierte Durchbrüche des Verfassungsvertrages, haben offenbar nicht die gleiche Überzeugungskraft wie Binnenmarkt oder Währungsunion, input-orientierte Legitimitätssteigerung nicht die gleiche Attraktivität wie das Versprechen eines konkreten outputs. Hinzu kommt, dass Schritte zur Etablierung eines am Maßstab der sozialen Marktwirtschaft gemessenen Gleichgewichts der beiden Komponenten zwar im Ersten Teil des Verfassungsvertrages unternommen werden, dass aber der Dritte Teil diesen Weg nicht weiter mitgeht.

Aus dieser freilich sehr verkürzten Analyse könnte sich jedenfalls als strategisches Integrationsziel für die deutsche Europapolitik in der Tat die Einlösung der Forderung nach einer sozialen Marktwirtschaft auf europäischer Ebene ergeben. Nach den großen Integrationsprojekten der letzten beiden Jahrzehnte, Binnenmarkt und Währungsunion im Bereich Vertiefung, europäische Einigung über die ehemaligen Blockgrenzen hinweg im Bereich Erweiterung, läge eine gewisse Logik darin, das Projekt, Soziale Marktwirtschaft' in Angriff zu nehmen. Wie immer man auch sich ein ,Weißbuch" mit einem Legislativprogramm zur Umsetzung dieses Leitbildes vorstellen mag, ein solches politisches Projekt würde die Lücke füllen, die im Verfassungsvertrag besteht, würde damit den Argumenten der Kritiker entgegenkommen, die Gründe für ihre ablehnende Haltung entkräften und stünde weder im Widerspruch zum Koalitionsvertrag noch zu dem Gebot der Konsensfähigkeit politischer Vorhaben innerhalb einer Großen Koalition in Deutschland. Es scheint, als sei beim EVPGipfel vor dem Europäischen Rat vom 15./16. Dezember in diese Richtung gedacht worden: Ein sozialpolitisches Projekt solle der Verfassung an die Seite gestellt werden, sozusagen zu ihrer Ergänzung, damit sie (auch in Frankreich und den Niederlanden) noch einmal und nun mit besseren Chancen zur Abstimmung gestellt werden könne. Ob und wann sich dieses Projekt konkretisiert ist freilich zur Zeit noch nicht absehbar. Sicher wird nicht anzuvisieren sein den redistributiven Teil der Sozialpolitik, sondern allenfalls den regulativen, auf europäische Ebene zu heben. Diese beiden Aspekte voneinander zu unterscheiden, zu klären, wie die Europäisierung des letzteren zu bewerkstelligen ist, wenn der erstere in nationalstaatlicher Regie verbleiben soll - das ist eine lohnende Aufgabe für Think-Tanks und Planungsstäbe, wenn denn das hier angedeutete Leitbild operationalisiert werden soll.

Allerdings wurde beim letzten Versuch, der in der CDU unternommen wurde, diese Leitbilddimension zu ergründen, eine Sozialunion für nicht wünschenswert erklärt - das ,Zweite Schäuble-Lamers-Papier‘, vom Mai 1999, war eine der ersten konzeptionellen Reaktionen auf die Vollendung der Währungsunion und antwortete schon auf die heute, nach erneutem Amtsantritt einer CDU-Kanzlerin akute Frage nach einem politischen Projekt angesichts der weitgehenden Ausschöpfung der marktwirtschaftlichen Integrationsdynamik. Schäuble und 
Lamers sprachen in diesem immer noch aktuellen Entwurf von einem „Wettbewerbsföderalismus", der ein hohes Maß an Marktintegration, dagegen aber einen Wettbewerb der Sozialsysteme, die in staatlicher Kompetenz bleiben sollten, vorsah - und schließlich wiederum ein hohes Maß an Integration im außen-, sicherheits- und verteidigungspolitischen Bereich.

Die zuletzt sich andeutende Variante eines Leitbildes für die nächste Integrationsepoche ist in der Tat die dritte, die der deutschen Europapolitik der nächsten Jahre lohnende Ziele setzen könnte. Ihr Charakteristikum wäre auch ein schwergewichtiges Integrationsprojekt, die Entwicklung einer wirklichen gemeinsamen Außenpolitik, einer Sicherheits- und Verteidigungsunion. Auch dieses Projekt stünde nicht im Widerspruch zur Kontinuität, trotz Schröderscher Alleingänge à la ,Deutscher Weg' sind von deutscher Seite bei Regierungskonferenzen und Konventsdebatten immer erhebliche Schritte zur Vergemeinschaftung dieser Politikbereiche bis hin zur Einführung von Mehrheitsentscheidungen verfochten worden. Einvernehmen unter den Koalitionspartnern müsste sich über ein derartiges Leitbild herstellen lassen. Zudem ist die Integrationsdynamik in den letzten zehn Jahren ohnehin wohl auf keinem anderen Gebiet so kräftig gewesen wie auf diesem. Christoph Heusgen, als außenund sicherheitspolitischer Berater der Kanzlerin, könnte durchaus in einem solchen Projekt eine besondere Attraktivität erkennen, zumal er selbst an der allseits mit Recht gelobten, prägnanten und anspruchsvollen Formulierung dieses Leitbildes - in Gestalt der Europäischen Sicherheitsstrategie von 2003 - maßgeblich beteiligt war. Auch die öffentliche Meinung in Europa steht stabil hinter einem solchen Ausbau der Union auf außen-, sicherheitsund verteidigungspolitischem Gebiet, auch mit diesem policy-Projekt würde man einen Hebel zur Durchsetzung des Verfassungsvertrages gewinnen - es sei daran erinnert, dass schon zu Beginn der 1950er Jahre die Einsicht vorhanden war, es müsse eine verfasste Politische Union geben, wenn eine Verteidigungsgemeinschaft Realität werden solle.

Dem Koalitionsvertrag konnte man vielleicht noch nachsagen, was in der CDU allgemein an Kritik am Wahlkampf formuliert wurde, dass nämlich beide, Wahlkampf und Koalitionsvertrag, zu pragmatisch, zu sehr auf das Nächstliegende fixiert, zu wenig leitbildorientiert waren. Jetzt, nach dem gelungenen Gipfel zur Finanziellen Vorausschau, nach dem über alle realistischen Erwartungen erfolgreichen Start in die Europapolitik, nun ist das Feld frei für eine ausgreifende politische Perspektive - mit dem Erfolg steigen bekanntlich die Ansprüche, auch an die deutsche Europapolitik: Die nächste Etappe könnte die Realisierung des Verfassungsvertrages sein, aber dieser Schritt sollte Teil eines lohnenden Integrationszieles, eines erneuerten Leitbildes für das Europa der Zukunft sein. 